\title{
Pancreatic hepatoid carcinoma: A case report and literature review
}

\author{
YU-YING LEI ${ }^{1 *}$, YAN-QIONG WANG $^{2 *}$, JIN-GANG HAO $^{3}$ and MING-WEI LIU ${ }^{4}$ \\ ${ }^{1}$ Intensive Care Unit, and ${ }^{2}$ Department of Anesthesiology, The First Affiliated Hospital of Kunming Medical University, \\ Kunming, Yunnan 650032; ${ }^{3}$ Department of Radiology, The Second Affiliated Hospital of Kunming Medical University, \\ Kunming, Yunnan 650106; ${ }^{4}$ Department of Emergency Medicine, The First Affiliated Hospital of \\ Kunming Medical University, Kunming, Yunnan 650032, P.R. China
}

Received May 8, 2021; Accepted August 4, 2021

DOI: $10.3892 /$ wasj.2021.120

\begin{abstract}
Hepatoid carcinomas (HCs) are a group of heterogeneous tumors that are similar to hepatocellular carcinoma (HCC). Pancreatic hepatoid carcinoma (PHC) is a very rare disease. The present study presents the case of a 63-year-old male patient with PHC with synchronous liver, spleen and stomach metastasis, in an aim to enhance our understanding of this disease. A 63-year-old male presented with abdominal distension for 3 months and slightly developed asthenia and anorexia. Laboratory tests of serum tumor markers revealed the alpha fetoprotein level was markedly elevated $(>10,00.0 \mathrm{ng} / \mathrm{ml})$. The radiological findings of computed tomography, magnetic resonance imaging, diffusion-weighted imaging and magnetic resonance cholangiopancreatography revealed a malignant tumor in the tail of the pancreas with synchronous liver, spleen, stomach and retroperitoneal lymph node metastasis. A post-operative immunohistochemical analysis revealed that the tumor cells in the tail of the pancreas exhibited positivity for glypican-3; right liver positivity was also observed for CK-18 and weak positivity for glypican-3, suggestive of primary PHC. On the whole, PHC is very rare, and exhibits morphological features similar to those of HCC. Its diagnosis is mainly achieved via morphological analysis and immunohistochemical staining of the tissue specimens, since the clinical manifestation and laboratory test results are often unspecific.
\end{abstract}

Correspondence to: Professor Jing-Gang Hao, Department of Radiology, The Second Affiliated Hospital of Kunming Medical University, 1 Mayuan Road, Wuhua, Kunming, Yunnan 650106, P.R. China

E-mail: 317325999@qq.com

Professor Ming-Wei Liu, Department of Emergency Medicine, The First Affiliated Hospital of Kunming Medical University, 295 Xichang Road, Wuhua, Kunming, Yunnan 650032, P.R. China E-mail: 1mw2004210@163.com

${ }^{*}$ Contributed equally

Key words: hepatoid carcinoma, pancreas, immunohistochemistry, morphology

\section{Introduction}

Hepatoid carcinomas (HCs) are a group of heterogeneous tumors that represent the morphology and immunohistochemistry of focal hepatocellular carcinoma ( $\mathrm{HCC}$ ), which comprises of pure forms of $\mathrm{HC}$ or a combination of other morphological features of acinar cell carcinomas, endocrine tumors or ductal adenocarcinomas. It has been reported in different primary sites of various cell origins, including the stomach, esophagus, lungs, gallbladder, urinary bladder, colon, ampulla of Vater, uterus, fallopian tubes, adrenal gland, ovaries, thymus, biliary tract, and other sites (1-16). Since Hruban et al (17) first reported primary pancreatic hepatoid carcinoma (PHC) in 1987, only 41 cases of primary PHC have been reported in the English literature, at least to the best of our knowledge $(4,17-52)$. The present study presents the case of patient with PHC with synchronous liver, spleen and stomach metastasis.

\section{Case report}

A 63-year-old male patient was transferred to the Second Affiliated Hospital of Kunming Medical University with a history of asthenia, anorexia and abdominal distension for a period of 3 months prior. A mass in the body and tail of pancreas, spleen, fundus and body of the stomach, and segments VI and VII of the liver was revealed upon magnetic resonance imaging (MRI) in an outside hospital over a 3-month period before presenting to the Second Affiliated Hospital of Kunming Medical University.

The patient had a history of hypertension for 2 years with a maximum blood pressure of 192/100 $\mathrm{mmHg}$. He also had a 30-year history of smoking and alcohol consumption. He denied a family history of cancer and inherited disease or any other specific personal history. The patient was found to have an ill-defined immobile solid mass $(\sim 6 \times 2 \mathrm{~cm})$ located in the epigastrium. The abdomen was soft, with no tenderness and rebound tenderness, and the patient had normal bowel sounds.

Laboratory examinations. Laboratory tests of serum tumor markers revealed that the alpha fetoprotein (AFP) level was markedly elevated $(>1,000.0 \mathrm{ng} / \mathrm{ml}$; reference range, at the 
Second Affiliated Hospital of Kunming Medical University is $0.0-8.0 \mathrm{ng} / \mathrm{ml}$ ) and the ferritin level was mildly decreased $(21.69 \mathrm{ng} / \mathrm{ml}$; reference range at the Second Affiliated Hospital of Kunming Medical University is 32.0-501.0 ng/ml). Furthermore, the levels of carcinoembryonic antigen (CEA), carbohydrate antigen 19-9 (CA19-9), neuron-specific enolase (NSE), carbohydrate antigen 50 (CA50) and carbohydrate antigen 724 (CA724) were within the normal range. The serum albumin, aspartate aminotransferase (AST) and alanine aminotransferase (ALT) levels were also within the normal range. The coagulation function tests and the analyses of serological markers for human immunodeficiency virus (HIV), hepatitis B virus (HBV) and hepatitis $\mathrm{C}$ virus (HCV) revealed negative results. However, the urine amylase level was markedly elevated (10,185 U/1; reference range at our hospital is 30.0-640.0 U/1).

Imaging examinations. A CT scan revealed that there were some ill-demarcated heterogeneous soft tissue density masses in the tail of pancreas, spleen and fundus of the stomach, respectively and in the right lobe of the liver; this was a mixed and low attenuation mass, measuring $8.9 \times 6.4 \mathrm{~cm}$.

Contrast-enhanced abdominal CT images revealed a mild-moderate enhancement, which had not only invaded the liver, but had also invaded the bottom of the stomach, spleen and retroperitoneal lymph node (Fig. 1). An MRI also revealed the presence of an ill-demarcated mass among the pancreatic tail and spleen, defined as isointense in the T1-weighted MR image and as mildly isointense-hyperintense in the T2-weighted MR image; the lesion had a size of $\sim 8.04 \times 10.46 \times 11.43 \mathrm{~cm}$. It was defined as hyperintense in diffusion-weighted imaging (DWI) and with a low apparent diffusion coefficient (ADC) value of $0.104 \pm 0.11 \times 10^{-3} \mathrm{~mm}^{2} / \mathrm{sec}$ ) (Figs. 2 and 3). Magnetic resonance cholangiopancreatography (MRCP) revealed that the hepatic portal bile duct and intrahepatic bile duct were invisible, and there were no abnormal findings in the extrahepatic bile duct and pancreatic duct. The radiological diagnostic impression was a malignant tumor in the tail of the pancreas with synchronous liver, spleen, stomach and retroperitoneal lymph node metastasis.

Treatment. Following comprehensive assessment, the patient underwent surgery, distal pancreatectomy with splenectomy, right hemihepatectomy, cholecystectomy, subtotal gastrectomy, gastroenterostomy and abdominal lymphadenectomy.

Pathological examination. The gross pathological analysis of the pancreatic resected specimen revealed a $6 \times 5 \times 4 \mathrm{~cm}$ gray-yellow tenacious mass involving the peripancreatic adipose tissue, with multiple suspicious lymph nodes. The pathological analysis of the live resected specimen revealed a $9 \times 8.5 \times 6.5 \mathrm{~cm}$ gray-yellow tenacious mass. The pathological analysis of the stomach also revealed the stomach wall adhesion with the spleen and pancreas, there was a rough $4.5 \times 4.5 \mathrm{~cm}$ 'discontinuation' of the mucosa, the cut section was a gray-yellow tenacious neoplasm, resembling cancer infiltration, the peristomachic adipose tissue had multiple lymph nodes that may exhibit metastasis. The pathological analysis of the spleen section revealed a $12 \times 9 \times 7 \mathrm{~cm}$ gray-yellow tenacious nodular neoplasm, the adhesion of the hilum of spleen with the pancreatic mass, and the presence of multiple lymph nodes that may exhibit metastasis. The histopathological report revealed a malignant tumor, which was defined as PHC which had metastasized to the liver, stomach, spleen and lymph nodes.

The immunohistochemical analysis (performed by the Clinical Laboratory of the Second Affiliated Hospital of Kunming Medical University,) of the tail of the pancreas revealed positivity for lower molecular weight cytokeratin (CKL) (data not shown), Villin(data not shown), glypican-3 (partially positive) and Ki-67 (40\%), whereas it revealed negative findings for hepatocyte-specific antigen (hepa-), CEA, vimentin, high molecular weight cytokeratin (CKH), CK-20, p53 and p63 (data not shown). The right liver was positive for CK-8 (data not shown), CK-18 (Fig. 4A), CD-34 (data not shown), glypican-3 (weak positivity; Fig. 4B) and Ki-67 (40\%), whereas it was negative for hepa-1, CK-7, CK-19 and CK-20 (data not shown). H\&E staining of the morphology of the tumor cells also revealed that the tumor cells were polygonal with large ovoid nuclei, prominent central nucleoli and an abundant eosinophilic cytoplasm (Fig. 4C).

Final diagnosis. The results of the morphological and immunohistochemical staining were consistent with an HC of the pancreas, with synchronous liver, spleen and stomach metastasis. The patient was then released from the hospital; at the time of discharge, the patient himself and his relatives provided consent for his data to be published; however, contact with the patient was lost 2 months after hospital discharge and thus a follow-up could not be performed.

\section{Discussion}

PHC is a very rare disease, and its diagnosis is mainly achieved through morphological analysis and immunohistochemical staining of the tissue specimens, since the clinical manifestation and the laboratory test results are often unspecific.

PHC exhibits morphological features similar to those of HCC, including large polygonal cells with an abundant eosinophilic cytoplasm and round nuclei, as well as prominent nucleoli in a trabecular or perisinusoidal pattern, occasionally featuring bile production and/or bile canaliculi formation. PHC has been previously reported in the literature in English, including 41 cases $(4,17-52)$, which can be divided into four histological subtypes: HC with pure HCC-like morphology, $\mathrm{HC}$ with neuroendocrine differentiation, and $\mathrm{HC}$ with acinar or glandular differentiation $[(4,18,33,53-54)$. As regards the pure HCC-like features, it has been differentially defined, including $\mathrm{HC}$, hepatoid adenocarcinoma, ectopic HCC, a hepatoid variant of pancreatic cancer, primary HCC of the pancreas or a pancreatic tumor with hepatoid differentiation (19).

The pathogenesis of PHC is not yet completely understood, although some hypotheses have been proposed. Some researchers have reported that the pancreas and liver all originate from the same area of primitive embryonic foregut in direct continuation with the yolk sac (55). It is possible that pancreatic cells retain the potential to differentiate into hepatic cells. As previously demonstrated, following the treatment of the rat pancreatic exocrine cell line, AR42J, with the synthetic glucocorticoid, dexamethasone, cells resembling hepatocytes are then induced (56). Furthermore, mature hepatocytes have the ability to differentiate into pancreatic cells (57). It has also been reported that the pancreas contains hepatocyte 

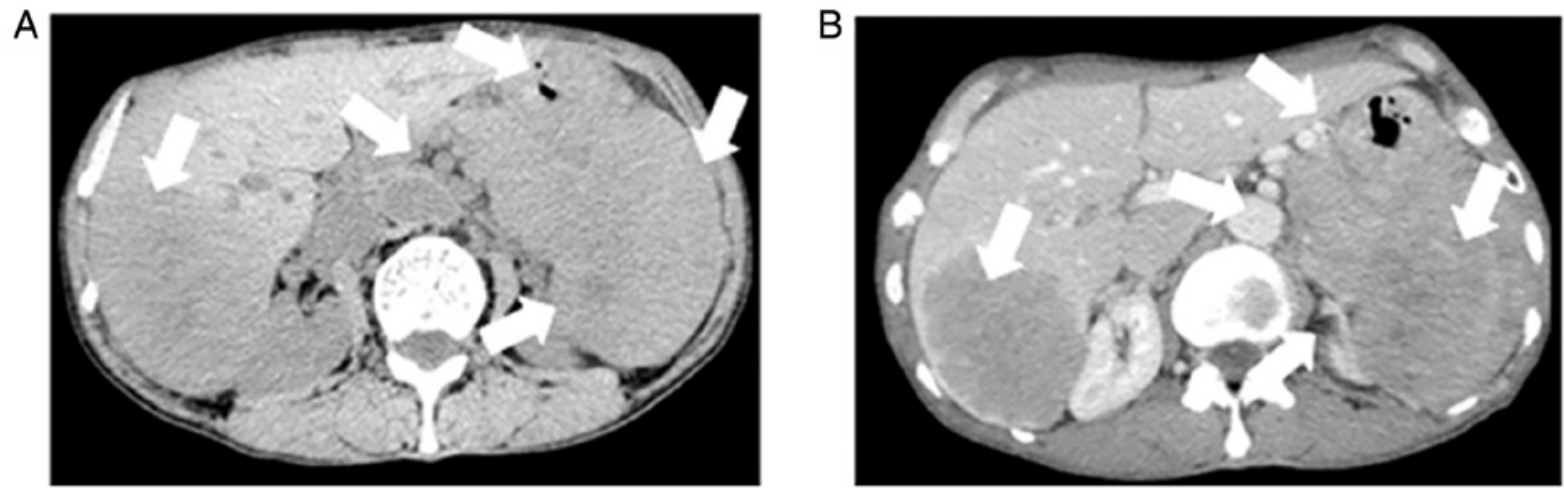

Figure 1. (A) An abdomen CT indicating an ill-demarcated heterogeneous mass located in the tail of the pancreas that invaded into the liver, stomach, spleen and retroperitoneal lymph node. A mixed and low attenuation right-liver mass is shown. The arrows indicate an ill-demarcated heterogeneous mass located in the tail of the pancreas that invaded into the liver, stomach, spleen and retroperitoneal lymph node. (B) Contrast-enhanced abdominal CT image showing a mild-moderate enhancement, findings suggestive of pancreatic cancer with synchronous liver, spleen, stomach and retroperitoneal lymph node metastasis. The arrows indicate pancreatic cancer with synchronous liver, spleen, stomach and retroperitoneal lymph node metastasis CT, computed tomography.
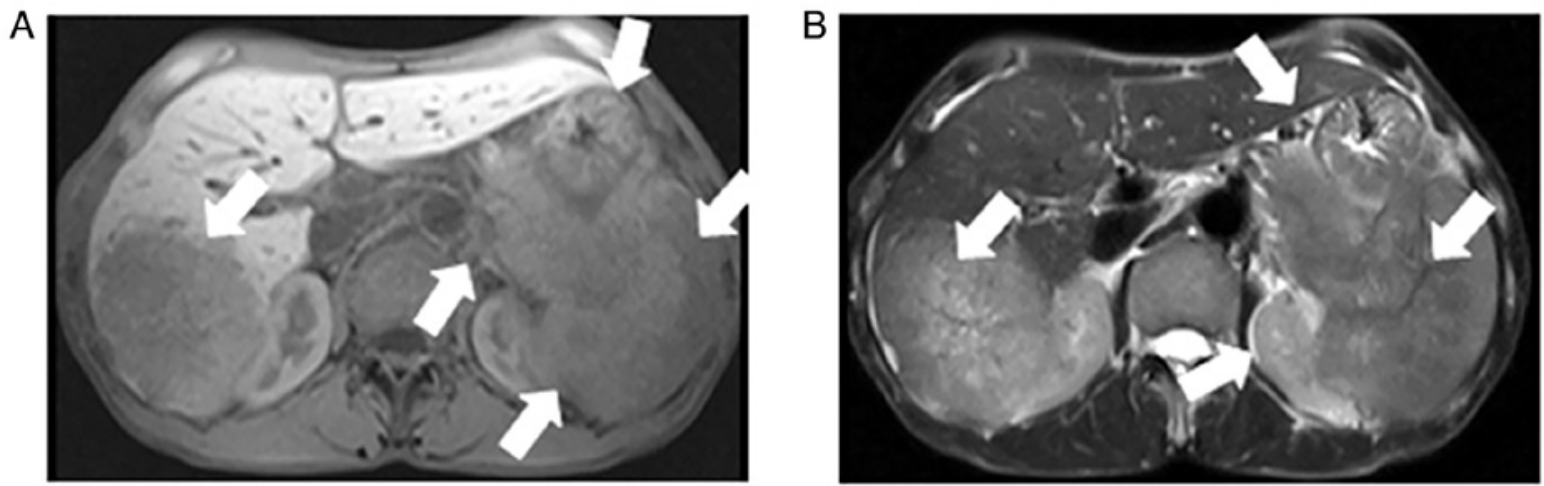

Figure 2. Abdominal MRI showing similar findings that are suggestive of a pancreatic cancer in the tail of pancreas with synchronous liver, spleen, stomach and retroperitoneal lymph node metastasis. (A) T1-weighted image and (B) T2-weighted image. MRI, magnetic resonance imaging. The arrows indicate a pancreatic cancer in the tail of the pancreas with synchronous liver, spleen, stomach and retroperitoneal lymph node metastasis.
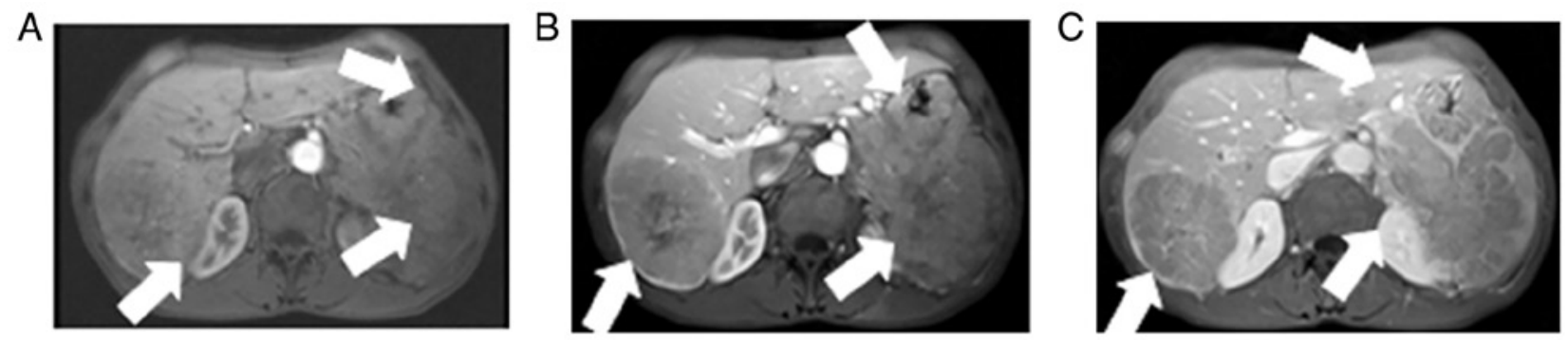

Figure 3. Abdominal dynamic contrast-enhanced showing (A) mild enhancement in the early arterial phase, (B) continuous mild washout in the portal phase and (C) later phase, with obvious enhancement in the capsular later phase. The lesion is pushing the distal right hepatic vein. The arrows indicate (A) mild enhancement in the early arterial phase, (B) continuous mild washout in the portal phase and (C) later phase, with obvious enhancement in the capsular later phase.

multipotent cells that have the ability for use in liver reconstruction therapy in adult mice (58). Therefore, it can be hypothesized that genes associated with hepatocyte differentiation are suppressed by pancreatic stem cells, which can be activated in a particular environment. Less than $5 \%$ of HCC cases arise in an ectopic area of liver tissue. Cardona et al (21) claimed that HC arose from ectopic liver tissue. Steen et al (22) considered that their patient's HCC arose in ectopic liver tissue located in the pancreas, and eventually replaced all original ectopic liver tissue. Tong et al (59) reported a case of hepatoid adenocarcinoma originating from a heterotopic pancreas in the ileum.

Serum AFP levels have been found to be elevated in the majority of cases of PHC (4,18,20,24-28,31,32,38,41,44-45,4 $7,52)$ similar to the case presented herein. Thus, AFP can be used to evaluate the integrity of surgical resection and response to therapies. However, it is not always specific to PHC. It is noteworthy that AFP levels may also be elevated in acinar cell carcinoma, as well as in ductal carcinoma of the pancreas, and in neuroendocrine, germ-cell tumors and undifferentiated 

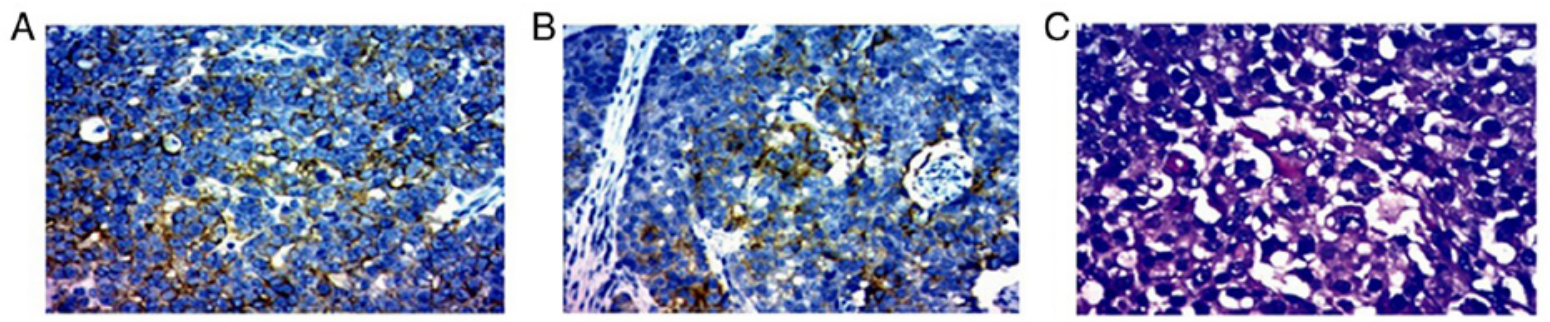

Figure 4. Pathological findings of the biopsy specimen of the pancreatic mass. Immunohistochemical staining showing the (A) CK-18 positive expression in specimen of liver and (B) glypican-3 positive expression in the pancreas tissue. Original magnification, $\mathrm{x} 200$. (C) Morphology of the tumor cells was polygonal with large ovoid nuclei, prominent central nucleoli and abundant eosinophilic cytoplasm (H\&E staining; original magnification, x200).

pancreatic adenocarcinoma $(60,61)$. PHC also shares immunohistochemical characteristics with HCC, such as AFP, Hep Parl, glypican-3, arginase-1, anti-albumin, anti- $\alpha$-1-antitrypisine, anti-low molecular weight cytokeratin, anti-epithelial membrane antigen antibodies and CD10. Polyclonal CEA stains exhibiting focal canalicular patterns in PHC have been reported in the English literature $(4,18,33)$. Due to the limited quantity of PHC cases reports in the literature, and PHC without specific performance in CT and MRI images, from the images, it cannot be distinguished whether this is a primary pancreatic tumor or metastatic HCC. In the case presented herein, the CT images revealed an ill-demarcated heterogeneous mass located in the tail of the pancreas that invaded the stomach, spleen and retroperitoneal lymph node; from the scans of the pancreas or stomach, a mixed and low attenuation right-liver mass was observed, suggestive of primary HCC, not excluding the metastatic lesions (Fig. 1A). Contrast-enhanced abdominal CT images revealed a mild-moderate enhancement (Fig. 1B). The MRI findings were suggestive of pancreatic cancer with synchronous liver, spleen, stomach and retroperitoneal lymph node metastasis (Fig. 2). There are no established diagnostic criteria available for PHC; however, without a doubt, these tumor marker stains play a vital role. Among these markers, Hep Parl seems to be the most sensitive marker for PHC (62). In the case in the present study, the tumor cells in the tail of the pancreas were positive for glypican-3 (Fig. 4B), and the right liver was positive for CK-18 (Fig. 4A) and weakly positive for glypican-3. There are some novel and more specific tumor markers for the diagnosis PHC reported, such as PIVKA-II, which was described by Matsueda et al (25) to be increased in a case of PHC in 2006. In addition, other immunohistochemical markers, such as bile salt export pump (BSEP) and multidrug-resistance protein 3 (MDR3) may be helpful for the diagnosis (61) Some researchers have revealed spalt-like transcription factor 4 (SALL4) staining in one third of $\mathrm{HC}$ cases (38).

During the diagnosis of primary PHC, metastatic HCC from the liver to the pancreas needs to be taken into consideration. Furthermore, other primary pancreatic tumors with an eosinophilic cytoplasm, such as neuroendocrine tumors, acinar cell carcinoma and solid pseudopapillary neoplasm should be distinguished $(20,27)$. These can be distinguished by their own histological characteristics and immunohistochemistry. Acinar cell carcinoma is negative for arginase-1 (62), whereas the reported poorly differentiated HCCs are positive for the marker (63-65). Hence, it is considered that arginase-1 seems to be a promising marker which can be used to discriminate poorly differentiated HCCs from acinar cell carcinoma. In addition, pancreatic neuroendocrine tumors are negative for glypican-3. Solid pseudopapillary neoplasms are often positive for glypican-3 (66).

Due to no unified standards available for treatment and according to recommendations from previous reports, that according to patients' tolerance, surgical resection is the preferred alternative for a disease-free survival. An early detection is also crucial $(29,60)$. A pervious study reported a case in which the oral multitarget tyrosine kinase inhibitor, sorafenib, with the function of promoting apoptosis and anti-angiogenesis, was used in the treatment of metastatic PHC (30). Ma et al (31) reported a case of PHC with liver metastasis treated with neoadjuvant modified-FOLFIRINOX (mFOLFIRINOX) chemotherapy followed by surgical resection; the patient's progression-free survival was 13 months. Other authors have described a case in which pancreatic neuroendocrine carcinoma was successfully treated with surgery followed by six cycles of post-operative chemotherapy (gemcitabine intravenously); the patient survival was 46 months (32). Pellini Ferreira et al (33) reported a case which was diagnosed as PHC with an increase in glucagon levels; treatment with octreotide intramuscular (IM) injections followed by chemotherapy was recommended with oral capecitabine and temozolomide; the patient ran a 10-mile marathon following 15 months of treatment. There are differential opinions regarding chemotherapy. Some authors recommend adjuvant chemotherapy, whereas others consider that a short-term regression can be achieved; however, no additional benefits have been found in the long-term $(31,39)$. Further studies and novel treatment methods are required in order to reveal the histobiological characteristics of PHC and its effectiveness and sensitivity to chemotherapy. Chang et al (34) first reported a case of $\mathrm{HC}$ with Caris Molecu-lar Intelligence ${ }^{\circledR}$ 600 gene sequencing; the mutational analysis revealed BAP1 and NOTCH1 with known alterations. The utilization of next-generation nucleic acid sequencing may help to determine the nature of genetic mutations and may help to reveal the detailed characteristics of this rare tumor and may also guide therapy. Next-generation profiling is a promising approach for rare tumors.

Due to its rarity and the lack of evidence-based data, the prognosis cannot be predicted precisely. PHC tends to have an aggressive outcome. It is considered that those with a pure HCC-like morphology have a better prognosis than those with neuroendocrine differentiation and glandular or acinar differentiation. In the majority of cases, metastases to the liver and lymph nodes have also been observed $(30,60)$. Early tumor cells formed between the lymphatic sinuses benefit 
cancer cell invasion and AFP inhibition immunoreaction (1). In the case presented herein, the patient presented with metastasis to the liver, stomach and spleen. According to limited reports, after 3 years, $50 \%$ of adult patients who are initially diagnosed usually succumb to the disease (60). In conclusion, although PHC is extremely rare, its characterization is essential.

In conclusion, PHC is very rare, and it is more easily diagnosed via morphological and immunohistochemical staining of the tissue specimens, as the clinical manifestation and the laboratory test results are often unspecific. Among these markers, Hep Par1 seems to be the most sensitive marker for PHC. Some novel tumor markers for the diagnosis PHC have been reported, such as PIVKA-II, BSEP and MDR3, which may prove to be helpful for the diagnosis. As there are currently no unified standards available for treatment, and in accordance with reports on the disease, the recommendation is as per patients' tolerance, surgical resection is the preferred alternative for disease-free survival. However, due to its rarity and the lack of evidence-based data, the prognosis of PHC cannot be predicted precisely. PHCs also tend to have an aggressive outcome and early detection is crucial.

\section{Acknowledgements}

The authors are grateful for the excellent assistance with the editing of the manuscript provided by Professor Lan-Fang Qin and Professor Xu Liu (Yan-an Hospital of Kunming, Kunming, China).

\section{Funding}

The present study received a research grant [grant no. 2017FE468(-032)] from the Yunnan Applied Basic Research Project-Union Foundation.

\section{Availability of data and materials}

The datasets used and/or analyzed during the current study are available from the corresponding author on reasonable request.

\section{Authors' contributions}

YYL and YQW participated in the conception and design of the study. MWL and JGH were also involved in the conception of the study and in the communications with the patient himself and his relatives. YYL and YQW prepared and completed the manuscript. MWL, JGH and YYL confirm the authenticity of all the raw data. All authors have read and approved the final manuscript.

\section{Ethics approval and consent to participate}

The study was approved by the Second Affiliated Hospital of Kunming Medical University and patient informed consent was obtained from the patient himself and his relatives.

\section{Patient consent for publication}

Consent for his data to be published was provided by the patient himself and his relatives at the time of discharge.

\section{Competing interests}

The authors declare that they have no competing interests.

\section{References}

1. Ishikura H, Fukasawa Y, Ogasawara K, Natori T, Tsukada Y and Aizawa M: An AFP producing gastric carcinoma with feature of hepatic differentiation: A case report. Cancer 56: 840-848, 1985.

2. Cappetta A, Bergamo F, Mescoli C, Lonardi S, Rugge M and Zagonel V: Hepatoid adenocarcinoma of the colon: What should we target? Pathol Oncol Res 18: 93-96, 2012.

3. Randolph LK, Hopkins MK, Hopkins MP and Wasdahl DA: Hepatoid carcinoma of the ovary: A case report and review of the literature. Gynecol Oncol Rep 13: 64-67, 2015.

4. Vanoli A, Argenti F, Vinci A, La Rosa S, Viglio A, Riboni R, Necchi V, Pugliese L, Sessa F, Pietrabissa A and Paulli M: Hepatoid carcinoma of the pancreas with lymphoid stroma: First description of the clinical, morphological, immunohistochemical, and molecular characteristics of an unusual pancreatic carcinoma. Virchows Arch 467: 237-245, 2015

5. Li M, Yang K and Wang P: Hepatoid adenocarcinoma of fallopian tube: A case report and review of the literature. Medicine (Baltimore) 98: e14534, 2019.

6. Devi NR, Sathyalakshmi R, Devi J and Lilly SM: Hepatoid adenocarcinoma of the gall bladder-A rare variant. J Clin Diagn Res 9: ED09-ED10, 2015.

7. Kato K, Suzuka K, Osaki T, Itami M and Tanaka N: Primary hepatoid adenocarcinoma of the uterine cervix. Int $\mathrm{J}$ Gynecol Cancer 17: 1150-1154, 2007.

8. Haninger DM, Kloecker GH, Bousamra Ii M, Nowacki MR and Slone SP: Hepatoid adenocarcinoma of the lung: Report of five cases and review of the literature. Mod Pathol 27: 535-542, 2014.

9. Kashani A, Ellis JC, Kahn M and Jamil LH: Liver metastasis from hepatoid adenocarcinoma of the esophagus mimicking hepatocellular carcinoma. Gastroenterol Rep (Oxf) 5: 67-71, 2017.

10. Sekino Y, Mochizuki H and Kuniyasu H: A 49-year-old woman presenting with hepatoid adenocarcinoma of the urinary bladder: A case report. Med Case Rep 7: 12, 2013.

11. Franke A, Ströbel P, Fackeldey V, Schäfer R, Göller T, Becker HP, Schöneich R, Müller-Hermelink HK and Marx A: Hepatoid thymic carcinoma: Report of a case. Am J Surg Pathol 28: 250-256, 2004.

12. Malya FU, Bozkurt S, Hasbahceci M, Cipe G, Ahmad IC, Gucin Z, Karatepe O and Muslumanoglu M: A rare tumor in a patient with hepatic hydatic cyst: Adrenal hepatoid adenocarcinoma. Case Rep Med 2014: 824574, 2014.

13. Takahashi N, Aoyama F, Hiyoshi M, Kataoka H and Sawaguchi A: Establishment and biological characterization of a novel cell line derived from hepatoid adenocarcinoma originated at the ampulla of Vater. Int J Oncol 44: 1139-1145, 2014.

14. Palas J, Ramalho M, Matos AP and Herédia V: Case 194: Periampullary hepatoid adenocarcinoma with duodenal invasion. Radiology 267: 959-963, 2013.

15. Wang Y, Liu YY and Han GP: Hepatoid adenocarcinoma of the extrahepatic duct. World J Gastroenterol 19: 3524-3527, 2013

16. Tomboravo C, Narindra LHRO, Ranoharison HD and Ahmad A: Hepatoid carcinoma of the pancreas: A case report. J Pancreat Cancer Treat 2: 6-9, 2019.

17. Hruban RH, Molina JM, Reddy MN and Boitnott JK: A neoplasm with pancreatic and hepatocellular differentiation presenting with subcutaneous fat necrosis. Am J Clin Pathol 88: 639-645, 1987.

18. Kai K, Nakamura J, Ide T, Masuda M, Kitahara K, Miyoshi A, Noshiro $\mathrm{H}$ and Tokunaga O: Hepatoid carcinoma of the pancreas penetrating into the gastric cavity: A case report and literature review. Pathol Int 62: 485-490, 2012.

19. Kuo PC, Chen SC, Shyr YM, Kuo YJ, Lee RC and Wang SE: Hepatoid carcinoma of the pancreas. World J Surg Oncol 13: 185, 2015.

20. Hameed O, Xu H, Saddeghi S and Maluf H: Hepatoid carcinoma of the pancreas: A case report and literature review of a heterogeneous group of tumors. Am J Surg Pathol 31: 146-152, 2007.

21. Cardona D, Grobmyer S, Crawford JM and Liu C: Hepatocellular carcinoma arising from ectopic liver tissue in the pancreas. Virchows Arch 450: 225-229, 2007.

22. Steen S, Wolin E, Geller SA and Colquhoun S: Primary hepatocellular carcinoma ('hepatoid' carcinoma) of the pancreas: A case report and review of the literature. Clin Case Rep 1: 66-71, 2013. 
23. Tanno S, Obara T, Fujii T, Izawa T, Mizukami Y, Saitoh Y, Ura H and Kohgo Y: Alpha-Fetoprotein-producing adenocarcinoma of the pancreas presenting focal hepatoid differentiation. Int J Pancreatol 26: 43-47, 1999.

24. Yano T, Ishikura H, Wada T, Kishimoto T, Kondo S, Katoh $\mathrm{H}$ and Yoshiki T: Hepatoid adenocarcinoma of the pancreas. Histopathology 35: 90-92, 1999.

25. Matsueda K, Yamamoto H, Yoshida Y and Notohara K: Hepatoid carcinoma of the pancreas producing protein induced by vitamin $\mathrm{K}$ absence or antagonist II (PIVKA-II) and alpha-fetoprotein (AFP). J Gastroenterol 41: 1011-1019, 2006.

26. Paner GP, Thompson KS and Reyes CV: Hepatoid carcinoma of the pancreas. Cancer 88: 1582-1589, 2000.

27. Kelly PJ, Spence R, Dasari BV, Burt AD, Taylor M and Loughrey MB: Primary hepatocellular carcinoma of the pancreas: A case report and review of the heterogeneous group of pancreatic hepatoid carcinomas. Histopathology 60: 1012-1015, 2012.

28. Lam K, Lo C, Wat M and Fan ST: Malignant insulinoma with hepatoid differentiation: A unique case with alpha-fetoprotein production. Endocr Pathol 12: 351-354, 2001.

29. Veerankutty FH, Yeldho V, Tu SA, Venugopal B, Manoj KS and Vidhya C: Hepatoid carcinoma of the pancreas combined with serous cystadenoma: A case report and review of the literature. Hepatobiliary Surg Nutr 4: 354-362, 2015.

30. Petrelli F, Ghilardi M, Colombo S, Stringhi E, Barbara C, Cabiddu M, Elia S, Corti D and Barni S: A rare case of metastatic pancreatic hepatoid carcinoma treated with sorafenib. J Gastrointest Cancer 43: 97-102, 2012.

31. Ma T, Bai X, Li G, Wei S and Liang T: Neoadjuvant modified-FOLFIRINOX followed by surgical resection of both the primary and metastatic tumors of a pancreatic hepatoid carcinoma with synchronous liver metastasis: A case report. Medicine (Baltimore) 96: e8413, 2017.

32. Xin BB, Li JA, Han X, Zhao J, Ji Y, Lou WH and Xu XF: Successful treatment of a case with pancreatic neuroendocrine carcinoma with focal hepatoid differentiation: A case report and literature review. Int J Clin Exp Med 7: 3588-3594, 2014.

33. Pellini Ferreira B, Vasquez J and Carilli A: Metastatic hepatoid carcinoma of the pancreas: First description of treatment with capecitabine and temozolomide. Am J Med Sci 353: 610-612, 2017

34. Chang JM, Katariya NN, Lam-Himlin DM, Haakinson DJ, Ramanathan RK, Halfdanarson TR, Borad MJ, Pannala R, Faigel D, Moss AA and Mathur AK: Hepatoid carcinoma of the pancreas: Case report, next-generation tumor profiling, and literature review. Case Rep Gastroenterol 10: 605-612, 2016.

35. Cuilliere P, Lazure T, Bui M, Fabre M, Buffet C, Gayral F and Bedossa P: Solid adenoma with exclusive hepatocellular differentiation: A new variant among pancreatic benign neoplasms? Virchows Arch 441: 519-522, 2002

36. Majumder $S$ and Dasanu CA: Hepatoid variant of pancreatic cancer: Insights from a case and literature review. JOP 14: 442-445, 2013.

37. Hughes K, Kelty S and Martin R: Hepatoid carcinoma of the pancreas. Am Surg 70: 1030-1033, 2004.

38. Yang C, Sun L, Lai JZ, Zhou L, Liu Z, Xi Y, Tao Y, Dooley E and Cao D: Primary hepatoid carcinoma of the pancreas: A clinicopathological study of 3 cases with review of additional 31 cases in the literature. Int J Surg Pathol 27: 28-42, 2019.

39. Williams NL, Palmer JD, Bar-Ad V, Anné PR, Sama AR, Weinstein JC, Rufail ML, Yeo CJ and Hurwitz MD: Hepatoid carcinoma of the pancreas: A case report and review of the literature. Case Reports in Pancreatic Cancer 1: 3-6, 2015.

40. Shih NN, Tsung JS, Yang AH, Tsou MH and Cheng TY: A unique pancreatic tumor with exclusive hepatocytic differentiation. Ann Clin Lab Sci 36: 216-221, 2006.

41. Oh HJ, Cheung DY, Kim TH, Kim SS, Kim MS, Kim JI, Park SH, Han JY, Han NI, Kim JK, et al: A case of hepatoid carcinoma of the pancreas. Korean J Gastroenterol 47: 389-393, 2006 (In Korean).

42. Kubota K, Kita J, Rokkaku K, Iwasaki Y, Sawada T, Imura J and Fujimori T: Ectopic hepatocellular carcinoma arising from pancreas: A case report and review of the literature. World J Gastroenterol 13: 4270-4273, 2007.

43. Liu CZ, Hu SY, Wang L, Zhi XT, Jin B, Zhu M, Wachtel MS and Frezza EE: Hepatoid carcinoma of the pancreas: A case report. Chin Med J (Engl) 120: 1850-1852, 2007.

44. Zhang Y,Zhou P and Sun Y: Hepatoid carcinoma of the pancreas: A case report. Chin J Clin Oncol 4: 445-447, 2007.

45. Jung JY, Kim YJ, Kim HM, Kim HJ, Park SW, Song SY, Chung JB, Kang CM, Pyo JY, Yang WI and Bang S: Hepatoid carcinoma of the pancreas combined with neuroendocrine carcinoma. Gut Liver 4: 98-102, 2010.
46. Huang SC, Chang HC, Yeh TS, Ng KF and Chen TC: Hepatoid microcarcinoma of the pancreas: A case report and review of the literature. Chang Gung Med J 35: 285-291, 2012.

47. Soofi Y, Kanehira K, Abbas A, Aranez J, Bain A and Ylagan L: Pancreatic hepatoid carcinoma: A rare form of pancreatic neoplasm. Diagn Cytopathol 43: 251-256, 2015.

48. Antonini F, Angelelli L, Rubini C and Macarri G: Endoscopic ultrasound diagnosis of a primary hepatoid carcinoma of the pancreas. Endoscopy 47 Suppl 1 UCTN: E367-E368, 2015.

49. Stamatova D, Theilmann L and Spiegelberg C: A hepatoid carcinoma of the pancreatic head. Surg Case Rep 2: 78, 2016.

50. Akimoto Y, Kato H, Matsumoto K, Harada R, Oda S, Fushimi S, Mizukawa S, Yabe S, Uchida D, Seki H, et al: Pancreatic hepatoid carcinoma mimicking a solid pseudopapillary neoplasm: A Challenging case on endoscopic ultrasound-guided fine-needle aspiration. Intern Med 55: 2405-2411, 2016.

51. Fukushima K and Kobayashi A: Hepatoid carcinoma of the pancreas mimicking neuroendocrine tumor. Hepatobiliary Surg Nutr 7: 501-502, 2018

52. Zeng SX, Tan SW, Fong CTH, Liang Q, Zhao BL, Liu K, Guo JX and Tao J: Hepatoid carcinoma of the pancreas: A case report and review of the literature. World J Clin Cases 8: 1116-1128, 2020.

53. Tomino T, Ninomiya M, Matono R, Narutomi $F$, Oshiro $Y$, Watanabe K, Taniguchi D, Nishimura S,Zaitsu Y,Kajiwara Y, et al: Pure pancreatic hepatoid carcinoma: A surgical case report and literature review. Surg Case Rep 5: 186, 2019.

54. Trinh HS, Luong TH, Lai TT and Nguyen TK: Mixed pancreatic hepatoid carcinoma: A surgical case report and literature review. Int J Surg Case Rep 83: 105951, 2021.

55. Ghurburrun E, Borbath I, Lemaigre FP and Jacquemin P: Liver and pancreas: Do similar embryonic development and tissue organization lead to similar mechanisms of tumorigenesis? Gene Expr 18: 149-155, 2018.

56. Eberhard D, O'Neill K, Burke ZD and Tosh D: In vitro reprogramming of pancreatic cells to hepatocytes. Methods Mol Biol 636: 285-292, 2010.

57. Reddy JK, Rao MS, Qureshi SA, Reddy MK, Scarpelli DG and Lalwani ND: Induction and origin of hepatocytes in rat pancreas. J Cell Biol 98: 2082-2090, 1984.

58. Makino T, Usuda N, Rao S, Reddy JK and Scarpelli DG: Transdifferentiation of ductular cells into hepatocytes in regenerating hamster pancreas. Lab Invest 62: 552-561, 1990.

59. Tong L, Pan H, He J, Weng M and Zheng L: Hepatoid adenocarcinoma arising from heterotopic pancreas of the ileum: A case report. Medicine (Baltimore) 95: e4067, 2016

60. Marchegiani G, Gareer H, Parisi A, Capelli P, Bassi C and Salvia R: Pancreatic hepatoid carcinoma: A review of the literature. Dig Surg 30: 425-433, 2013.

61. Fujikura K, Yamasaki T, Otani K, Kanzawa M, Fukumoto T, $\mathrm{Ku}$ Y, Hirose T, Itoh T and Zen Y: BSEP and MDR3 useful immunohistochemical markers to discriminate hepatocellular carcinomas from intrahepatic cholangiocarcinomas and hepatoid carcinomas. Am J Surg Pathol 40: 689-696, 2016.

62. Askan G, Deshpande V, Klimstra DS, Adsay V, Sigel C, Shia J and Basturk O: Expression of markers of hepatocellular differentiation in pancreatic acinar cell neoplasms: A potential diagnostic pitfall. Am J Clin Pathol 146: 163-169, 2016.

63. Radwan NA and Ahmed NS: The diagnostic value of arginase-1immunostaining in differentiating hepatocellular carcinoma from metastatic carcinoma and cholangiocarcinoma as compared to HepPar-1. Diagn Pathol 7: 149, 2012.

64. Fujiwara M, Kwok S, Yano H and Pai RK: Arginase-1 is a more sensitive marker of hepatic differentiation than HepPar-1 and glypican-3 in fine-needle aspiration biopsies. Cancer Cytopathol 120: 230-237, 2012

65. Yan BC, Gong C, Song J, Tretiakova M, Hyjek E, Al-Ahmadie H, Alves V, Xiao SY, Anders RA and Hart JA: Arginase-1: A new immunohistochemical marker of hepatocytes and hepatocellular neoplasms. Am J Surg Pathol 34: 1147-1154, 2010.

66. Hav M, De Potter A, Ferdinande L, Van Bockstal M, Lem D Eav S, Pattyn P, Praet M, Cuvelier C and Libbrecht L: Glypican-3 is a marker for solid pseudopapillary neoplasm of the pancreas. Histopathology 59: 1278-1279, 2011.

his work is licensed under a Creative Commons Attribution-NonCommercial-NoDerivatives 4.0 International (CC BY-NC-ND 4.0) License. 